\title{
CORRIGENDUM
}

\section{Mitogenic Sonic hedgehog signaling drives E2F1-dependent lipogenesis in progenitor cells and medulloblastoma}

\author{
B Bhatia ${ }^{1}$, M Hsieh $^{2}$, AM Kenney ${ }^{1}$ and Z Nahlé
}

${ }^{1}$ Department of Cancer Biology and Genetics, Memorial Sloan-Kettering Cancer Center, New York, NY, USA and ${ }^{2}$ Department of Cardiothoracic Surgery, Weill Cornell Medical College, New York, NY, USA

Oncogene (2011) 30, 504; doi:10.1038/onc.2010.524

Correction to: Oncogene (2011) 30, 410-422; doi:10.1038/ onc.2010.454; published online 4 October 2010

After the article was published online, the author list and affiliations were found to be incorrectly linked. The correct links between the authors and affiliations are shown above. 